\title{
Tracheal Atresia
}

National Cancer Institute

\section{Source}

National Cancer Institute. Tracheal Atresia. NCI Thesaurus. Code C35754.

A rare, fatal congenital malformation in which the trachea is severely underdeveloped. 